\title{
Influence of manufacturing methods on the homogeneity and properties of nitinol tubular stents
}

\author{
D. Favier, L. Orgéas, D. Ferrier ${ }^{1}$, P. Poncin ${ }^{1}$ and Y. Liu ${ }^{2}$ \\ Laboratoire Sols, Solides, Structures, UMR 5521 du CNRS, UJF-INPG, BP. 53, \\ 38041 Grenoble cedex, France \\ ${ }^{1}$ Société Minitubes SA, 7 avenue du Grand Chatelet, 38000 Grenoble, France \\ 2 Department of Mechanical and Materials Engineering, University of Western Australia, \\ Nedlands, WA 6009, Australia
}

\begin{abstract}
Nitinol has found growing applications in vascular stents, with the recent introduction on the market of a number of new nitinol tubular devices for coronary and peripheral usage. These stents can be produced either through the expansion of a pattern cut on a small tube (pre-cut) or by cutting the deployed design on a tube of a larger size (pre-expanded) [1]. The aim of the study was to analyze the differences of properties induced by the two manufacturing methods. For this purpose, Differential Scanning Calorimetry (DSC) measurements were performed on various specimens from the two types of stents. A first set of measurements shows that pre-expanded stents exhibit usual $A \leftrightarrow R \leftrightarrow M$ transformations whereas multi-stage transformations occur for pre-cut stents. Two types of DSC specimens were then prepared for each stent, corresponding to the straight sections and the curved parts of the stent. It is shown that the pre-cut stents exhibit less homogeneous thermomechanical properties of the material within each stent compared to the pre-expanded stents. This is attributed to the manufacturing method which involves non-homogeneous bending stress-state for the pre-cut stents.
\end{abstract}

\section{INTRODUCTION}

Nitinol has found growing applications in vascular stents, with the recent introduction on the market of a number of new nitinol tubular devices for coronary and peripheral usage. These devices are produced from nitinol tubing in which mesh-like pattern is laser-cut or etched. Two recent papers $[1,2]$ have analyzed the influence of the manufacturing process, particularly of the choice of tubing size on which the pattern is cut. This choice affects the thermomechanical properties of the device, the cost and residual effects of laser cutting, and device design possibilities.

The aim of the present paper is to analyze the influence of the manufacturing process on the material properties in a stent. For this purpose, Differential Scanning Calorimetry (DSC) was performed to characterize the difference in thermomechanical behavior between stents fabricated by different manufacturing processes as well as the homogeneity of thermomechanical properties inside each stent.

\section{EXPERIMENTAL DESIGN AND SET-UP}

\subsection{Manufacturing of the stents}

The usual way to manufacture self-expandable slotted tube stents is to start with a tube of dimensions close to the compressed size of the stent. This folded design is laser-cut and the stent with its deployed dimensions is obtained by a succession of expansion/heat treatment at temperatures of the order of $500^{\circ} \mathrm{C}$ $(773 \mathrm{~K})$. These progressive shape settings lead to the final shape of the so-called "pre-cut stent". An alternate manufacturing method uses tubes with dimensions close to the deployed size of the so-called "pre-expanded stent". The deployed strut design is then laser-cut and a single heat treatment is applied.

In the present study, both types of stents were prepared for comparison. They were fabricated of the "standard superelastic Ti-50.8at\% Ni alloy". Pre-cut and pre-expanded stents were manufactured from tubes of diameters $2 \mathrm{~mm}$ and $10 \mathrm{~mm}$, respectively. 


\subsection{DSC apparatus and specimens}

The transformation behavior was analyzed by DSC measurement carried out on a 2920 TA Instruments Differential Scanning Calorimeter, with a heating/cooling rate of $10 \mathrm{~K} / \mathrm{min}$. Measurements were performed on specimens from manufactured stents and from tubes. For the stents, three specimens were prepared using small segments cut from each stent. The first specimens contained a mixture of segments whereas the second ones contained segments from the straight sections and the third ones contained segments from the curved sections. DSC specimens from tubes were cut using a low speed diamond cutoff wheel.

\section{EXPERIMENTAL RESULTS}

\subsection{Comparison of the two types of stents}

Fig.1 shows DSC measurements for one pre-expanded stent and for two pre-cut stents. The pre-expanded stent exhibits two transformation stages on cooling, from austenite to the $R$-phase, $A \rightarrow R$, and then to martensite, $\mathrm{R} \rightarrow \mathrm{M}$. The characteristic temperatures, as measured at the maximum heat flow on DSC curves, are labeled $\mathbf{R}$ - and $\mathbf{M}$ in Fig.1. The reverse transformation on heating is a two-step $M \rightarrow R \rightarrow A$ transformation whose characteristic temperatures are labeled $\mathbf{A}$ and $\mathbf{R}+$ in Fig.1.

In comparison, the pre-cut stents, while being highly reproducible with their transformation behavior, exhibit three stages of transformation on cooling and three stages of transformation on heating, as evident in Fig.1. The $A \rightarrow R$ transformation temperature, marked $R-$, is slightly higher and the martensitic transformation is split into two reaction stages. This multi-stage transformation has been reported in the literature to be induced by ageing treatment of near equiatomic NiTi alloys [3-6]. For the pre-cut stents, the peaks have been identified as $A \rightarrow R$ transition and two martensitic transformations $R \rightarrow M 1$ and $\mathrm{R} \rightarrow \mathrm{M} 2$ on cooling and two reverse transformations $M 2 \rightarrow \mathrm{R}$ and $\mathrm{M} 1 \rightarrow \mathrm{R}$ preceding the $\mathrm{R} \rightarrow \mathrm{A}$ transition on heating. The characteristic temperatures are labeled $\mathrm{R}-, \mathrm{M} 1, \mathrm{M} 2$ on cooling and $\mathrm{A} 2, \mathrm{~A} 1$ and $\mathrm{R}+$ on heating.

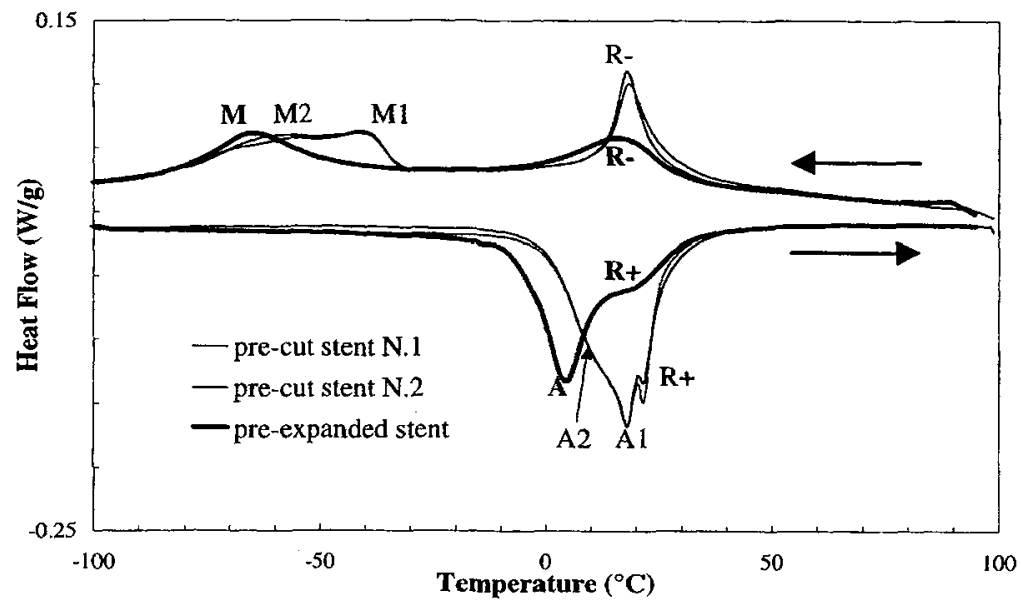

Figure 1: DSC for 2 pre-cut stents and one pre-expanded stent

\subsection{Comparison of transformation behavior of stents with that of their originating tubes}

Fig. 2 shows DSC measurements for a pre-expanded stent and for a tube of $10 \mathrm{~mm}$ in diameter on which pre-expanded stents were cut. Fig. 3 shows similar measurements for a pre-cut stent and for a tube of $2 \mathrm{~mm}$ in diameter on which the folded design for pre-cut stents was cut. It is seen that both the small and large tubes and the pre-expanded stent exhibit a two-step $\mathrm{A} \rightarrow \mathrm{R} \rightarrow \mathrm{M}$ transformation on cooling and a two-step $\mathrm{M} \rightarrow \mathrm{R} \rightarrow \mathrm{A}$ transformation on heating. The three-step transformations are only observed for the pre-cut stent. 


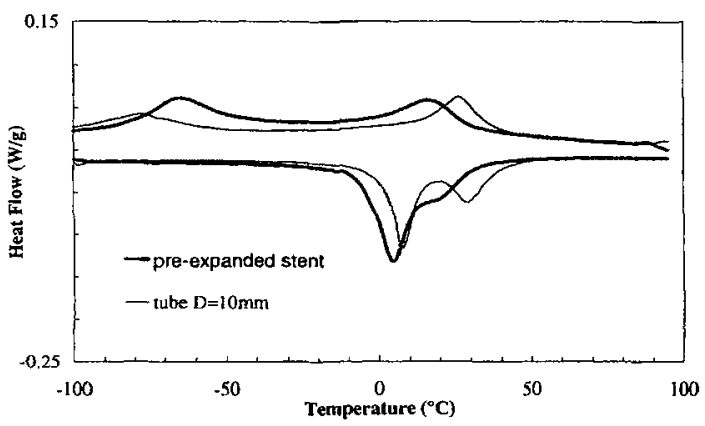

Figure 2: DSC for a pre-expanded stent and a large tube

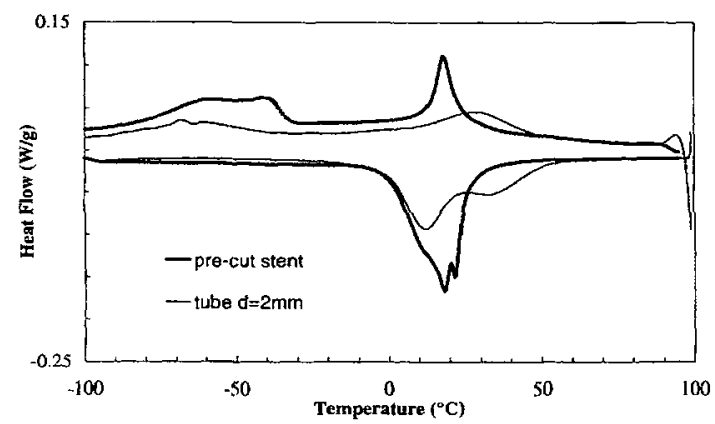

Figure 3: DSC for a pre-cut stent and a small tube

\subsection{Characterization of homogeneity inside each type of stent}

Fig.4 a shows DSC measurements for a pre-expanded stent for both straight sections and curved parts. Negligible difference is seen between the two specimens as evident in the enlarged portion of the curves shown in Fig.4.b. The two specimens show identical transformation behavior during heating, exhibiting partially overlapped $\mathrm{M} \rightarrow \mathrm{R}$ and $\mathrm{R} \rightarrow \mathrm{A}$ transformations.

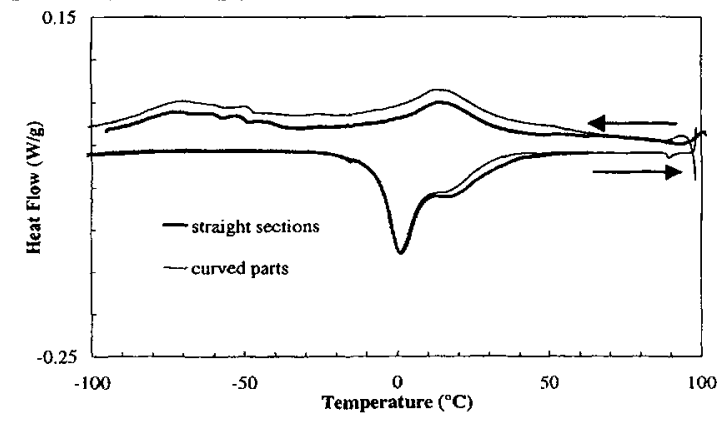

Figure 4.a: DSC for the straight and curved strut segments of a pre-expanded stent

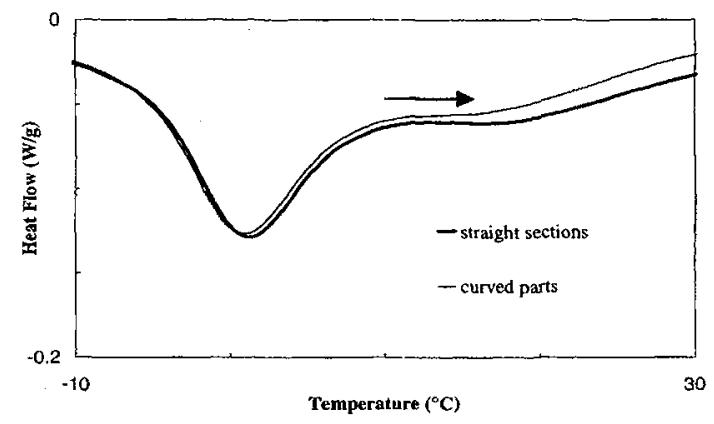

Figure 4.b: Enlargement of figure 4.a during heating

Fig.5.a shows similar measurements for a pre-cut stent. Differences in transformation behavior between the two specimens were observed. This observation is consistent for all pre-cut stents. It is seen in the enlarged portion of the curves for heating transformation, as shown in Fig.5.b, that the straight strut segments exhibited a more pronounced three-stage transformation behavior compared to the curved parts for the pre-cut stent.

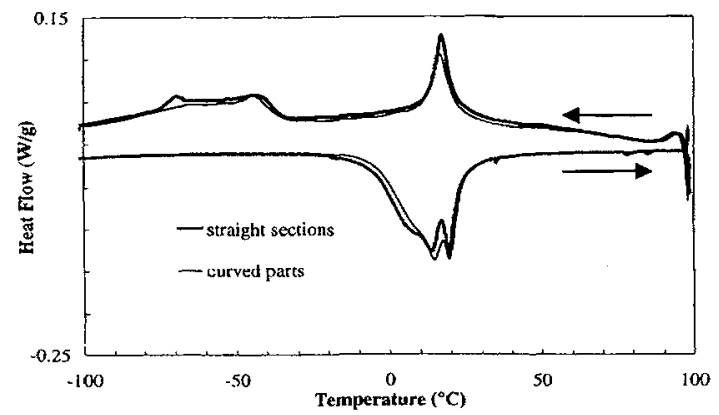

Figure 5.a: DSC for the straight and curved strut segments of a pre-cut stent

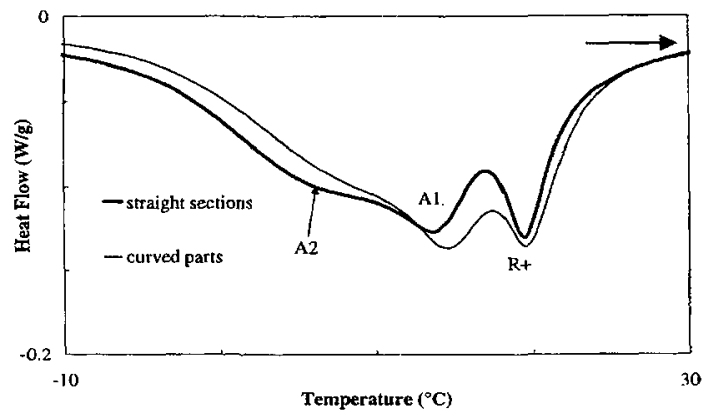

Figure 5.b: Enlargement of figure 5.a during heating 


\subsection{Development of multi-stage transformation for the NiTi used}

Small tubes of $2 \mathrm{~mm}$ in diameter were aged under different conditions. Fig.6 shows the DSC measurement of the effect of ageing treatment on transformation behavior. The specimen from an asreceived tube shows a complete austenite to R-phase transition. No clear peak on further cooling is recognized for the $\mathrm{R}$-phase to martensite transformation. However, on heating the specimen exhibited two peaks corresponding to $\mathrm{M} \rightarrow \mathrm{R}$ and $\mathrm{R} \rightarrow \mathrm{A}$ transformations, successively. An ageing at $500^{\circ} \mathrm{C}$ for $300 \mathrm{~s}$ results in the $A \leftrightarrow R$ transformation shifting to lower temperatures. This shift is increased by a second period of ageing under the same conditions. This additional ageing also leads to the occurrence of a threestep martensitic transformation, both on cooling and on heating. The first peak appearing on cooling, marked R-, corresponds to the appearance of the R-phase. The two following peaks, marked M1 and M2, reveal the successive transformation of the R-phase into two distinct martensites.

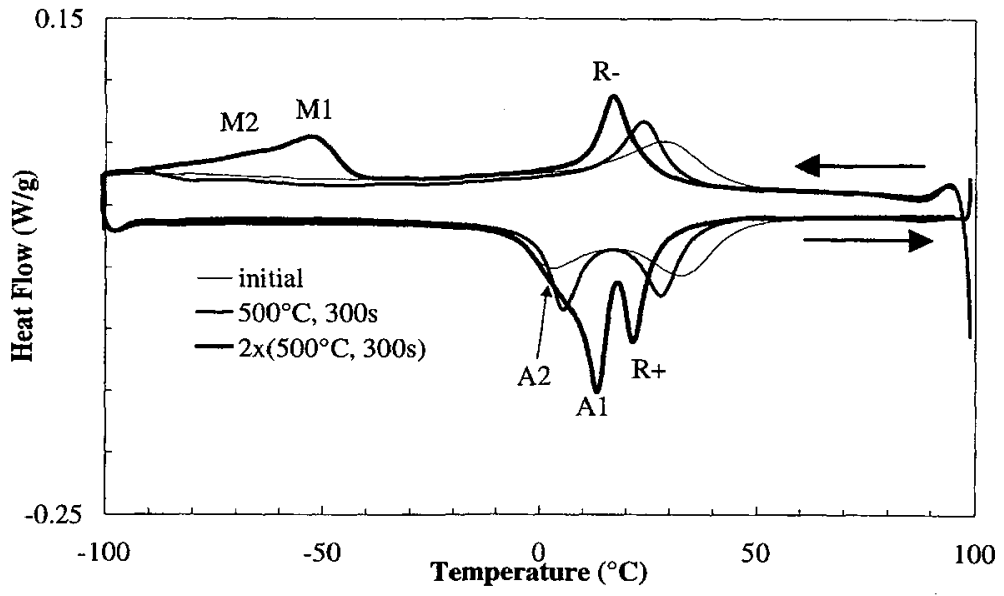

Figure 6: DSC measurements on aged small tubes

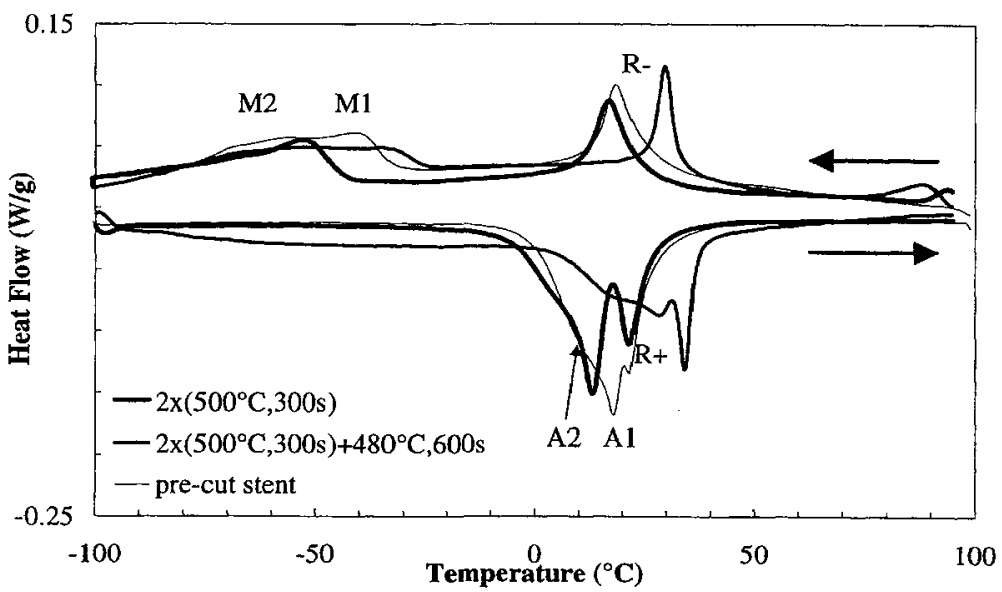

Figure 7: DSC measurements on aged small tubes and on a pre-cut stent

Fig.7 shows the influence of further ageing on the three-step martensitic transformation. An additional ageing at $480^{\circ} \mathrm{C}$ for $500 \mathrm{~s}$ is performed after the two first ageing treatments. After this ageing treatment, both the $A \leftrightarrow R$ transformations (marked $R$ - and $R+$ on cooling and heating, respectively) and the $R \leftrightarrow M 1$ transformations (marked Ml on cooling and $A 1$ on heating) are seen to shift to higher 
temperatures. There is a less change to the characteristic temperatures of $\mathrm{M} 2$ and $\mathrm{A} 2$ transformations. As a result, the three-stage aspect of the transformations is enhanced. The DSC curves for a pre-cut stent, also plotted in Fig.7, exhibit similar three-step transformations with characteristic temperatures appearing intermediate between those observed for tubes submitted to the two previous ageing treatments.

\section{DISCUSSION}

\subsection{Influence of the manufacturing process on the transformation}

The multi-stage transformation has been reported in the literature for $\mathrm{NiTi}$ alloys subjected to ageing treatment [3-6]. The results presented in $\$ 3.4$ on the effect of ageing on the transformation behavior for the tubes agree with the results of previous studies on Ni-rich nitinol (i.e. $>50.5$ at\% Ni). Several hypotheses have been proposed for this unusual transformation behavior. Favier et al. [4] attributed this phenomenon to ageing-induced precipitates. They suggested that elastic internal stress fields created by coherent precipitates locally assist the formation of martensites adjacent to the precipitates, whereas regions away from precipitate particles are not directly affected. Bataillard et al. [6] later provided transmission electron microscopic evidences demonstrating the effect of local stress fields around $\mathrm{Ni}_{4} \mathrm{Ti}_{4}$ precipitates on the multi-stage martensitic transformation behavior of a $\mathrm{Ti}-51.14 \mathrm{at} \% \mathrm{Ni}$ alloy aged at $520^{\circ} \mathrm{C}$, in support of the precipitate hypothesis for Ni-rich alloys. This hypothesis, however, has not been confirmed by microstructural analysis for near equiatomic NiTi alloys $(<50.2 \mathrm{at} \% \mathrm{Ni})$ [7].

For pre-expanded stents, a single heat treatment is required to stress-relieve the material after the laser cutting operation. This heat treatment is usually too brief to cause precipitation, hence to induce the multi-stage transformation, as shown in Fig.2. The expansion of the pre-cut stent requires multiple heat treatments, which cause precipitation. This is believed to be the reason for the multi-stage transformation exhibited by the pre-cut stents, as shown in Fig.3.

\subsection{Non-homogeneity in the pre-cut stent}

(a)

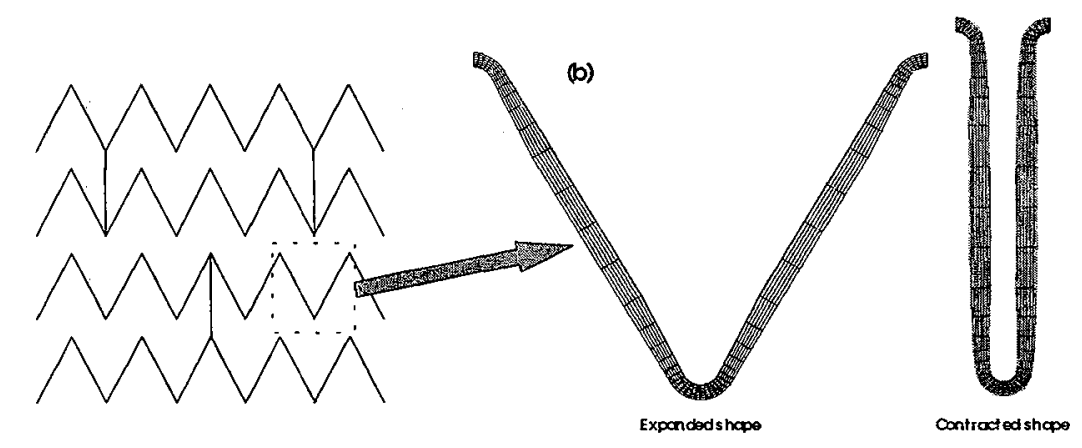

(c)

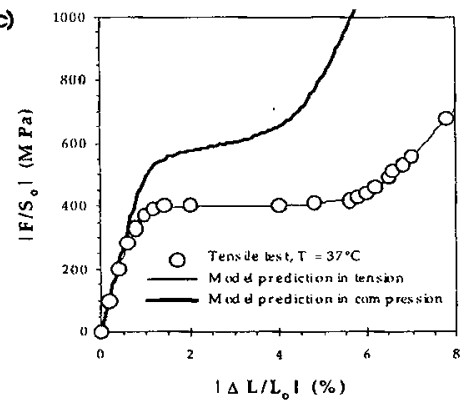

(d)

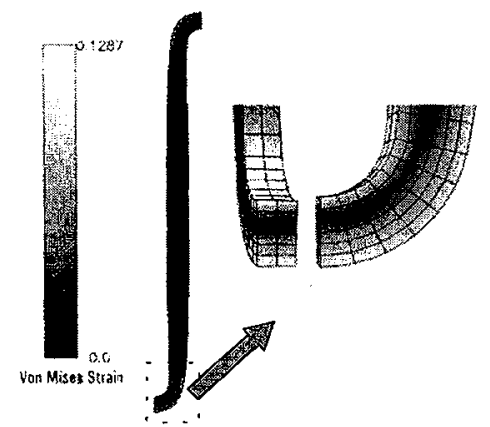

Figure 8: Finite element simulation of the expansion/contraction of a stent; (a): a stent considered as a periodic repetition of a simple motif; (b): contraction of one motif; (c): tensile tests at $37^{\circ} \mathrm{C}$ and prediction of the proposed constitutive equation in tension and compression; (d): simulated strain field in one half of a motif 
It is established that the ageing-induced multi-stage transformation behavior in Ni-rich alloys is due to the precipitates and the local stress fields associated with them [6]. All the previous works on the multi-stage transformation studied the effect of ageing with no applied external stress. It is known that external stresses affect the creation and shape of precipitates and of the local stress fields induced by ageing [8]. Finite element simulation [9] has been performed to estimate the stress and strain fields developed during the shape setting of a pre-cut stent. Fig. 8 shows results of such a simulation. The stent is considered as formed by the periodic repetition of a simple motif constituted of two straight struts linked by a curved one (Fig.8.a). Simulation has been performed on one simple motif to model the strain field induced by the contraction/expansion (Fig.8.b). Experiments in tension have been performed on the NiTi alloy at $37^{\circ} \mathrm{C}$ and a constitutive equation has been fitted on these experimental results, as shown in Fig.8.c. The proposed constitutive equation takes into account the non-symmetric behavior observed in tensioncompression for the superelastic deformation of $\mathrm{NiTi}$ alloys [10]. Simulation shows that, during the expansion of the stent, the straight segments experience negligible strains and stresses, as shown in Fig.8.d. The enlargement shows that the strain state in the curved portion of the stent is highly nonhomogeneous. Due to the small radius of the curved region and to the non-symmetric tensioncompression behavior, the unstressed neutral axis is not in the middle of the thickness. The neutral axis is closer to the compression side, resulting in more material being in tension than in compression. With the used geometry and constitutive equations, the maximum strain for the complete expansion of the pre-cut stent is estimated to be higher than $12 \%$ in compression and higher than $10 \%$ in tension.

The ageing conditions are thus highly different through the stent during the expansion/heat treatment. It is suggested that the less pronounced three-stage behavior observed for the curved strut segments of the pre-cut stent (see Fig.5) could be due to the tension or compression stress state experienced by the material situated far from the neutral axis during the heat treatment. These additional external stresses are effective during ageing; it is suggested that these stresses could alter the internal stress development during the precipitation process. Experimental studies are in progress in order to verify this hypothesis.

\section{CONCLUSION}

Stents can be manufactured either from small and large tubes. This paper analyzed the influence of the manufacturing process on the properties of stents fabricated of the standard superelastic Ti-50.8at\% Ni alloy. DSC measurements were performed on stents manufactured by the two routes. DSC measurements on specimens from stents cut on a large tube exhibited a two-stage transformation behavior $A \leftrightarrow R \leftrightarrow M$ with a good homogeneity of the thermomechanical properties of the material within each single stent. For stents expanded from a small tube, all DSC specimen showed a three-stage transformation behavior, both during cooling and heating. This unusual ageing-induced behavior in Ni-rich alloys is due to the precipitates and the local stress fields associated with them. Moreover, this three-stage transformation behavior was less pronounced for the curved strut segments of the stents compared to the straight segments. It is suggested that this reduction is due to the influence of the macroscopic stresses experienced by the curved regions of the stents during the expansion procedure of the stents.

\section{References}

1. P.Poncin, D.Ferrier, C.Meyer-Kobbe, Proceedings of the $4^{\text {th }}$ Int. Conf. on ShapeMemory and Superelastic Technologies, (1999)

2. P.Poncin, D.Ferrier, A.Loshakove, J.Proft, C.Meyer-Kobbe, $5^{\text {th }}$ Int. Conf. on Shape Memory and Superelastic Technologies, Santa-Clara, California, USA, 2000, edited by A.Pelton, (2000)

3. J.S. Zhu, R. Gotthardt, Phys. Lett. A 132, 279 (1988).

4. D. Favier, Yinong Liu, P.G. McCormick, Scripta Metall. Mater. 28, 669 (1993).

5. L. Bataillard, R. Gotthardt, J. de Phys. IV 5, C8-647 (1995).

6. L. Bataillard, J.-E. Bidaux, R. Gotthardt, Philosophical Magazine A 78, 327 (1998).

7. Yinong Liu, X. Chen, P.G. McCormick, J. Materials Sci. 32, 5979 (1997).

8. M. Nishida, T. Honma, Scripta Metall. 18, 1293 (1984).

9. L.Orgeas, D.Favier, Revue Européenne des Eléments Finis, Vol.7, N.8, 111 (1999).

10. L.Orgeas, D.Favier, Acta Mat., Vol.46, N.15, 5579 (1998). 